\title{
Ethics in Medical Research and Publication
}

\author{
Khalid Tabbara, * M. Zuheir Al-Kawi †
}

$\mathrm{R}$ esearch in medicine is an activity conducted with the intent to produce new knowledge. Research may be basic or clinical, a retrospective analysis of data or a prospective clinical study. Research fosters new knowledge and improves clinical practice by improving diagnostic and therapeutic strategies. It is the medical activity that aims at the future. If necessity is the mother of invention, planning and hard work are its parents. In medical research, the scientific method is applied to an incredible diversity of issues. Evidence-based medicine requires that the results of research in prospective clinical trials be used as the best basis for management of many disorders.

In general, an observation followed by complex well-organized research confirms or refutes a theory. An erroneous impression fostered by sensational popular ideas is that scientific discovery is often made by inspiration or a coup-de-foudre style invention. Curiosity is an important prerequisite for finding solutions and is a requirement in research. Inventions are usually the final assimilation of innumerable facts and impressions by the mind of a clinician or scientist. Clinical problems give the impetus to research projects. Creative thinking, curiosity, and imagination give momentum to research. The scientist takes up from the manifold observations of his predecessors when carrying out a literature review. Reproduction of research may confirm a scientific observation, while innovation generates new knowledge. New discoveries may originate by serendipity. It is important to appreciate a clue, interpret it and later exploit it. Sir Alexander Fleming in 1928 at St. Mary's Hospital in London noticed that one culture plate got contaminated with a fungus and the colonies of Staphylococcus around the fungus died out. Following the lead of that significant observation, Fleming went on to discover penicillin, the first and most important antibiotic. This ultimately earned him the Nobel Prize.

One important aspect of our profession is its adherence to a code of ethics. Since its earliest recorded history, medical professionals have set specific standards of conduct to guide the behavior of members of the profession. The roots of medical ethics are in the moral and religious principles of the society. Conformity to these standards led to the higher status of the physician in society and safeguarded the welfare of patients.

Research has to be based on ethics and mutual trust between the investigator and the research participant. The declaration of Helsinki required that clinical trials should be performed only on volunteers. Innovative therapeutic approaches should always be in the patient's best interest and risks to patients are minimized. The risk/benefit ratio should be low to justify the research effort. Following Institutional Review Board (IRB) approval, research participants must be informed about the nature of the research. Consent is obtained and alternate therapeutic modalities should be explained and offered to the patient.

Ethical guidelines for public announcement of research may not be very clear to scientists. In the era of multimedia, rapid communication
From the *The Eye Center and The Eye Foundation for Research in Ophthalmology, Riyadh and the + Department of Neuroscience, King Faisal Specialist Hospital and Research Centre, Riyadh

Correspondence and reprint requests:

Khalid F. Tabbara, $M D$

FRCOphth, $A B O$ The Eye Center and The Eye Foundation for Research in Ophthalmology 241 Makkah Road

P.O. Box 55307 Riyadh 11534 Saudi Arabia Tel: +966-1-4649614 Fax: +966-1-4629675 k.tabbara@nesma.net.sa Ann Saudi Med 2006;26(4):257-260 
and public interest in new modalities of diagnosis and management, it has become commonplace for investigators to give public announcements of new developments in their research. Although it is vital for the media to obtain factual information about scientific developments, announcements must be presented in a language that is less liable to misinterpretation or wild extrapolation beyond what the investigator had intended. An announcement of preliminary results is often labeled as a "breakthrough" for public consumption and sensationalism. Besides creating false hopes and expectations, such announcements can backfire, resulting in loss of credibility in the scientific community as a whole. Carefully chosen language must be incorporated with assessing the risk/benefit ratio of such an announcement.

In medical research we face many ethical issues: proprietary interest, financial gain, personal recognition and promotion. Accountability, honesty and integrity are the pillars that maintain the validity of our research activities. ${ }^{1}$ It is unfortunate to hear about breach of honesty and integrity in medical research. Fraudulent papers with fabricated data or inflated results have appeared in the literature. ${ }^{2}$ The intense pressure on scientists and academician to publish or lose their grants and jobs may have fostered some cases of fraud in research.

Ethical issues pertaining to embryonic stem cell research assumed an important platform with scientists, politicians, and theologists. ${ }^{3}$ Recently, Hwang and associates reported a breakthrough on patientspecific embryonic stem cells derived from human stem cell nuclear transferred blastocysts. ${ }^{4}$ Seoul National University in South Korea, where Hwang works, concluded that he fabricated the data of two papers published in the journal Science in 2004 and 2005. This dealt a devastating blow to the reputation of Dr. Hwang as a pioneer in the field of embryonic stem cells. It was an embarrassment to the scientist and his institution and created public mistrust in other embryonic stem cell research done elsewhere. The journal Science announced on January 12, 2006, a retraction of both papers. Prosecutors barred his travel and raided his home.

Rules and regulations and codes and bylaws cannot replace individual conscience, honesty, and integrity. The trustworthy system of science stems from the moral integrity of the scientist. It is also the responsibility of the medical profession to ensure verification of the safety and efficacy of any new medical or surgical treatment. The basic principle underlying research is honesty. Each scientist has the responsibility to provide research results of high standards, to keep impeccable records, to interpret results correctly, to gather facts meticulously, and to report new knowledge through an appropriate scientific forum. Financial gain, professional promotion, public acclaim, and personal recognition should not be the primary motivation for carrying out scientific research. Co-authors should make a significant contribution to the research project and must be acquainted with the research work. All the investigators involved in a research project should have contributed to the study and attest to its integrity and validity. Recipients of research funds who provide support to the project should be active in the project.

Writing the manuscript is the art of science, and scientists publish papers out of interest in research, advancement and knowledge, and love of science. They should not have a conflict of interest. Pharmaceutical firms support some research projects and may have access and control of the investigators' data. They may elect not to publish data with negative results that may affect the sale of a product. On the other hand, the pharmaceutical company may pay the physician a professional advisory fee, support his research project, or pay his travel and accommodation to meetings. The investigator may possess stocks in a company. Such a relationship between scientists and pharmaceutical companies should be transparent and has to be acknowledged in presentations and published work. Underreporting of clinical trials can cover up the adverse effects of an intervention.., 6

The International Committee of Medical Journal Editors and the World Health Organization have endorsed the registration of clinical trials. This would require that details of a clinical trial be publicly disclosed before recruitment of patients as a condition for subsequent publication. Major medical journals such as Nerw England Journal of Medicine, JAMA, and the Annals of Internal Medicine, now require clinical trial registration. As of March 1, 2006, the Archives of Ophthalmology, American Journal of Ophthalmology, British Journal of Ophthalmology, Investigative Ophthalmology and Visual Science, and Ophthalmology will require that human clinical trials be registered prior to recruitment, to mention only a few.

The International Committee of Medical Journal Editors (ICMJE) has set criteria for authorship of papers submitted to biomedical journals. Authorship credit should be based on (1) substantial contributions to the conception and design or acquisition of data or analysis and interpretation of data, (2) 
drafting the article or revising it critically for important intellectual content, and (3) final approval of the version to be published. Authors should meet all three conditions. Acquisition of funding, collection of data, or general supervision of a research group does not justify authorship. All persons designated as authors should qualify for authorship and all those who qualify should be listed. Each author should have participated sufficiently in the work to take public responsibility for an appropriate portion of the content.

It should be recognized that individuals who perform clinical services to patients as part of their routine work are not co-authors of a scientific paper unless they have contributed significantly to the research project. ${ }^{7}$ Authors of scientific papers must make a significant and substantial contribution to the research project. The contribution can be in the design of the study, acquisition of data analysis and interpretation of data, writing of manuscript and approval of the final version of the manuscript. The contributions should be carefully defined.

It has been a tradition at certain institutions to include the name of the senior faculty member or chairman of the department in the published research work though he/she has not contributed to the project, to the analysis of the data, or to the writing of the manuscript. This practice represents a form of unjustified deception and mockery of the authorship of scientific papers, a practice that should be discouraged.

Plagiarism, on the other hand, represents usurping of intellectual property and is unethical. Incorporating the statements of others, either verbatim or through paraphrasing without attribution is unacceptable in the scientific community. Some authors may make modifications, add patients, and re-publish the papers to gain an extra publication. Recycling of papers is now easily discovered by internet search engines and may raise questions about the integrity of the author. Omission of data or inaccurate data presentation is also unacceptable.

In conclusion, research is a highly complex intellectual activity, especially in medicine. Medical professionals should conduct research to unravel the mysteries of diseases, replace speculation with fact, and improve diagnostic, medical and surgical management of diseases. The research should always abide by a code of ethics of the medical profession, protect the interest of the patients and the community, and avoid bias. We have the responsibility to encourage clinical and basic research in medicine

\section{Glossary of definitions and explanatory remarks}

- Plagiarism: Copying from published or unpublished manuscript, article or chapter without attribution or including exact wording of text or copying of tables or figures without permission from the publisher.

- Fabrication/falsification of data: Creation of data without proper experimentation or changing the numbers to improve the results. This is a form of fraud.

- Redundant publication: Utilization of data from another article and incorporation in a new format for a new article. This is also referred to as self-plagiarism.

- Duplication of publication: Repeat publication or publication of the same work in a different journal or forum. Occasionally, an article may be a chapter in another book or symposium.

- Gift authorship: Inclusion of names of persons who did not contribute to the intellectual effort and should be discouraged.

- Patient's rights: Patients included as research subjects should be willing volunteers, who have freely consented for participation after being informed about the nature of research, its potential risks, and alternative management.

- Conflict of interest: When authors have an interest (other than scientific) in promoting a product that was a subject in the publication. Such interest may be funding support by a pharmaceutical company or serving in an advisory role in the company or commercial entity. All such interests should be disclosed by the authors.

- Humans subjects rights: Investigators should adhere to the Declaration of Helsinki, Belmont report, and good clinical practice guidelines by the International Conference on Harmonization (ICH).

- Animals rights: Treatment of animals should follow guidelines that prevent cruelty to animals.

- Registration of Clinical Trials: All clinical trials must be registered prior to initiation. Most international peer-reviewed journals reject studies of clinical trials that have not been registered. 
within the code of medical ethics. Each institution should develop a means to monitor activities of its scientists, check the data of each experiment, and identify the type and degree of participation of each co-investigator.

The Kingdom of Saudi Arabia has established a National Committee of Biological and Medical Ethics. The committee in turn has developed detailed guidelines that were distributed. It continues to tackle issues pertaining to ethics in medical and biological research. ${ }^{8}$ Institutions involved in research should develop mechanisms and regulations that ensure compliance with the national committee's guidelines, and establish sufficient safeguards for protection of participants. Adhering to such rules maintains the confidence and respect that the society places in the medical profession.

\section{References}

1. Nakayama T, Sakai M Slingsby BT Japan's ethical guidelines for epidemiologic research a history of their development. J Epidemiol 2005;15(4):107-112.

2. Brouillet $M$, Turner $L$. Bioethics, religion, and democratic deliberation: policy formation and embryonic stem cell research. HEC Forum 2005:17(1):49-63.

3. Newell FW. Fabricated data and co authors. Am
J Ophthalmol 1987:103:101-2

4. Hwang WS, Roh SI, Lee BC, et al. Patient-specif ic embryonic stem cells derived from human SCNT blastocysts. Science 2005;308:1777-83.

5. Henry DA, Kerridge IH, Hill SR, McNeil PM Doran $E$, et al. Medical specialists and pharmaceutical industry-sponsored research: a survey of the Australian experience. Med J Aust 2005;182(11):549-50.
6. Freeman WR. Control of data, authorship, and responsibility for clinical trials publications (editorial). Ophthalmology 2005;112(9):1485-6.

7. Tabbara KF. Authorship of scientific manuscripts. Ann Saudi Med 1988;8:390.

8. National Committee on Bio and Medical Ethics http://bioethics.kacst.edu.sa 